\title{
RELACIÓN ENTRE EL GEN Lr67 DE RESISTENCIA A ROYA DE LA HOJA Y EL GEN Rht-D1 DE ENANISMO DEL TRIGO
}

\section{RELATIONSHIP BETWEEN THE Lr67 LEAF RUST RESISTANCE GENE AND THE Rht-D1 DWARFING GENE OF WHEAT}

\section{Magda K. Moedano-Mariano', Julio Huerta-Espino², Susanne Dreisigacker ${ }^{3}$, Héctor E. Villaseñor-Mir², Amalio Santacruz-Varela' ${ }^{1}$ Ricardo Lobato-Ortiz ${ }^{1}$ e Ignacio Benítez-Riquelme ${ }^{1}$ *}

\begin{abstract}
'Colegio de Postgraduados, Postgrado en Recursos Genéticos y Productividad-Genética, Montecillo, Texcoco, Estado de México, México. ${ }^{2}$ Instituto Nacional de Investigaciones Forestales, Agrícolas y Pecuarias, Campo Experimental Valle de México, Chapingo, Estado de México, México. ${ }^{3}$ Centro Internacional de Mejoramiento de Maíz y Trigo, Texcoco, Estado de México, México.
\end{abstract}

*Autor de correspondencia (riquelme@colpos.mx)

\section{RESUMEN}

El gen Lr67, localizado en el cromosoma 4D, confiere resistencia a la roya de la hoja causada por Puccinia triticina E. Este gen fue común en las variedades de trigo (Triticum spp.) de porte alto liberadas en México hasta antes de 1960, no así en las variedades semienanas liberadas desde esa fecha hasta el presente. Las variedades semienanas de trigo liberadas en México poseen ya sea el gen de enanismo Rht-B1 localizado en el cromosoma 4B 0 el gen Rht-D1 localizado en el cromosoma 4D. Con el fin de investigar si existe algún impedimento genético para conjuntar los genes Lr67 y Rht-D1 en la misma variedad de trigo, se caracterizó genotípicamente la $\mathrm{F}_{3}$ de la cruza entre la variedad Nasma que posee el gen Rht-D1 con la variedad Marroqui 588 poseedora del gen $L r 67$. Mediante pruebas de ji-cuadrada y marcadores moleculares de diagnóstico, se encontró que la distancia genética entre ambos genes es de $23.6 \mathrm{cM}$ con análisis de ligamiento y de $27.6 \mathrm{cM}$ con análisis molecular. Estas distancias son suficientemente grandes para que exista recombinación genética, por lo que no hay ningún impedimento genético para obtener genotipos recombinantes que combinen el gen de resistencia a roya de la hoja (Lr67) con el gen de porte bajo de planta (Rht-D1), mismos que en los progenitores se encuentran en fase de repulsión.

Palabras clave: Triticum aestivum, distancia genética, gen Lr67, gen Rht-D1, recombinación.

\section{SUMMARY}

Gene Lr67, located on chromosome 4D, confers resistance to leaf rust caused by Puccinia triticina $\mathrm{E}$. This gene was common in tall wheat (Triticum spp.) varieties released in Mexico before 1960, but not in semi-dwarf varieties released from that date to the present. Semi-dwarf wheat varieties released in Mexico carry either the Rht-B1 dwarfing gene located on chromosome 4B or the Rht-D1 gene located on chromosome $4 \mathrm{D}$. To test whether there is a genetic impediment to combine the Lr67 and Rht-D1 genes in the same wheat variety, the $\mathrm{F}_{3}$ of the cross between the Nasma variety, Rht-D1 gene carrier, and the Marroqui 588, Lr67 gene carrier, was genotypically characterized. By using chi-square tests and diagnostic molecular markers, it was found that genetic distance between both genes is $23.6 \mathrm{cM}$ with linkage analysis and $27.6 \mathrm{cM}$ with molecular analysis. These distances are large enough for genetic recombination to exist; thus, there is no genetic impediment to obtain recombinant genotypes that combine the leaf rust resistance gene ( $L r 67)$ with the dwarfing gene (Rht-D1), that in the parents are in repulsion phase.
Index words: Triticum aestivum, gene Lr67, gene Rht-D1, genetic distance, recombination.

\section{INTRODUCCIÓN}

La producción mundial de trigo (Triticum spp.) es afectada por plagas y enfermedades que reducen el rendimiento y la calidad del grano; entre las enfermedades, las royas son las que históricamente han causado las mayores pérdidas económicas, a pesar de los esfuerzos para obtener cultivares con resistencia genética (Rodríguez-García et al., 2019; Sandoval-Sánchez et al., 2019) y del uso intensivo de productos químicos (Singh et al., 2016).

De las tres royas que atacan al trigo: de la hoja (Puccinia triticina E.), del tallo ( $P$. graminis $f$. sp. tritici) y amarilla ( $P$. striiformis f. sp. tritici), la primera es la enfermedad con mayor adaptación a diversos ambientes y está ampliamente distribuida en el mundo; su presencia reduce el rendimiento desde $7 \%$ hasta más del $50 \%$ debido a que provoca un menor peso de grano, dependiendo en la etapa fenológica del cultivo en que se presente la infección (Huerta-Espino et al., 2011b).

La resistencia a las royas que presentan comúnmente los genotipos de trigo es conocida como de raza específica; sin embargo, los patógenos causantes de estas enfermedades pueden vencer dicha resistencia por su continua evolución hacia nuevos biotipos o nuevas razas fisiológicas, además de su rápida reproducción y capacidad de moverse a distancias muy grandes (Singh et al., 2016). También se ha buscado la resistencia en planta adulta (APR por sus siglas en inglés), que a menudo es de raza no específica y su uso y aplicación se considera una estrategia más confiable y duradera para combatir esta enfermedad (Li et al., 2016). Para generar variedades que posean genes APR que 
confieren resistencia horizontal se requiere sumar a varios genes en el genotipo (Niederhauser et al., 1954), genes que, si bien por sí solos no confieren la resistencia adecuada, especialmente bajo una presión alta de la enfermedad, cuando están juntos y en combinaciones piramidales de cuatro o cinco pueden conferir casi inmunidad o un alto nivel de resistencia (Singh et al., 2000).

Entre los genes que confieren resistencia de raza no específica a la roya de la hoja del trigo se han identificado cuatro con efectos significativos, mismos que también muestran efectos pleiotrópicos, ya que el mismo gen confiere resistencia a otras enfermedades. Los genes Lr34/ Yr18/Sr57/Pm38/Stb1/Ltn1, Lr46/Yr29/Sr58/Pm39/Ltn2, Sr2/Yr30/Lr27/Pbc1 y Lr67/Yr46/Sr55/Pm46 confieren resistencia a múltiples enfermedades como roya de la hoja, roya amarilla y cenicilla polvorienta (Blumeria graminis f. sp. tritici) (Herrera-Foessel et al., 2014).

El gen Lr67, que confiere resistencia a roya de la hoja en planta adulta, fue identificado en Canadá a partir de la introducción PI250413 de Pakistán (Dyck y Samborski, 1979) y se ubica en el cromosoma $4 \mathrm{DL}$ del trigo (Hiebert et al., 2010); además, provee resistencia parcial a la roya amarilla, designado como Yr46 (Herrera-Foessel et al., 2011), a la roya del tallo, nombrado Sr55 y resistencia a la cenicilla polvorienta, denominado Pm46 (Herrera-Foessel et al., 2014) y otras enfermedades foliares. También es fenotípicamente similar al gen de resistencia a roya de hoja Lr34 (Hiebert et al., 2010).

El gen Lr67 es muy común en variedades de porte alto como Marroqui 588, Chapingo 48, Yaqui 50, entre otras, liberadas en México y desarrolladas hasta antes de los años 1960s (Moore et al., 2015); sin embargo, está ausente en variedades semi-enanas modernas, tanto en las que poseen el gen de enanismo Rht1 o Rht-B1, como en las que poseen Rht2 o Rht-D1. En México, estos genes de enanismo están presentes en variedades semi-enanas que desde los años 1960s han permitido la producción de variedades con alto potencial de rendimiento y resistencia a enfermedades (Huerta et al., 2011a). Las variedades de trigo liberadas para el noroeste de México poseen el gen de enanismo Rht-B1, mientras que Rht-D1 fue más común en las variedades liberadas para El Bajío (Huerta et al., 2011a). Todas las variedades liberadas actualmente en México poseen ya sea Rht-B1 o Rht-D1, pero no ambos, excepto la variedad Oasis F86 liberada en 1986, que posee Rht2 (Sayre et al., 1997).

Hasta la fecha no se ha encontrado, entre todas las variedades de trigo harinero liberadas en México, alguna que combine a los genes $L$ r67 y Rht-D 1 ; el hecho de que no existan estas variedades sugiere un posible impedimento genético ocasionado por ligamiento cromosómico.

La ubicación de los genes Lr67 y Rht-D1 en el genoma del trigo es en el cromosoma 4DL. Por su ubicación en el mismo cromosoma y brazo, se supone entonces, que ambos genes no son independientes y que, por el origen de los progenitores, ambos genes están en fase de repulsión. El objetivo de esta investigación fue determinar la magnitud del ligamiento y con ello dilucidar la posibilidad de obtener individuos recombinantes que conjunten la resistencia que otorga el gen Lr67 con el de enanismo que confiere Rht-D1, bajo la hipótesis de que la distancia entre ambos genes en el cromosoma 4DL es suficientemente grande para permitir la recombinación entre ellos.

\section{MATERIALES Y MÉTODOS}

\section{Material genético}

Se utilizaron como fuentes de germoplasma las variedades Nasma, portadora del gen de enanismo Rht-D1, y a uno de sus progenitores, Marroqui 588 (= Florence) Aurore C). La variedad Marroqui 588, portadora del alelo Lr67 y de porte alto, es una cruza realizada en 1920 en Francia y liberada como variedad en Marruecos y Túnez (Jlibene y Nsarellah, 2011).

Se realizaron cruzamientos entre ambas variedades en el ciclo de cultivo de otoño/invierno 2013-2014 para obtener la generación $F_{1}$ en el ciclo primavera/verano 2014, en el Campo Experimental Valle de México del Instituto Nacional de Investigaciones Forestales, Agrícolas y Pecuarias (INIFAP-CEVAMEX). La semilla de tres plantas $F_{1}$ dieron origen a la $F_{2}$ que se sembró en el Campo Experimental Bajío del INIFAP en el ciclo de cultivo otoño/invierno 2014 2015, donde se cosecharon plantas individuales que generaron 296 familias $F_{3}$ evaluadas en el ciclo de cultivo primavera/verano 2015 en el INIFAP-CEVAMEX.

\section{Análisis molecular}

El análisis molecular se llevó a cabo en el laboratorio de Biotecnología del Programa de Mejoramiento Molecular del Trigo en el Centro Internacional de Mejoramiento de Maíz y Trigo (CIMMYT). Los progenitores Nasma y Marroqui 588 y cada familia de la generación $F_{3}$ se evaluaron con marcadores SNP para el gen Lr67 (csSNP856) y el gen Rht-D1 (Rht-D1_SNP). Simultáneamente, los progenitores se evaluaron con marcadores STS (Sitios de Secuencia Etiquetada) con el propósito de determinar la presencia de los genes de interés en ambos progenitores; se usó el marcador csLV67 para el gen Lr67, con la muestra vegetal RL6077 como control positivo y Avocet como control negativo (Moore et al., 2015), además del marcador DF/MR2 
para Rht-D1, con la muestra vegetal Parula como control positivo y Tonichi como control negativo (Ellis et al., 2002); en ambos casos se utilizó un marcador Phiø174 para peso molecular. Para el análisis de ADN, se colectaron pequeñas porciones del tejido foliar y se colocaron en tubos de $1.1 \mathrm{~mL}$ en placas de 96 pozos, se llenó hasta la mitad de cada tubo; posteriormente, se almacenaron en un congelador a -80 ${ }^{\circ} \mathrm{C}$ por $3 \mathrm{~h}$ y se llevaron a un liofilizador (Labconco, Kansas City, Missouri, EUA) a temperatura de $-50{ }^{\circ} \mathrm{C}$ y un nivel de vacío de 0.0 a 0.120 mBars por 48 h. El tejido se maceró con balines de $4 \mathrm{~mm}$ en un molino Geno/Grinder 2010 (Spex SamplePrep, Metuchen, New Jersey, EUA) por 2 a 3 min hasta obtener el tejido en polvo. La extracción, cuantificación y valoración de la calidad del ADN se realizó de acuerdo con los procedimientos descritos por Dreisigacker et al. (2016a).

El programa para la PCR (Kompetitive Allele Specific PCR genotyping system- KASPar) se ejecutó con la temperatura de amplificación más favorable de acuerdo con los protocolos del CIMMYT (Dreisigacker et al., 2016a). La lectura de las placas del producto de PCR se realizó en lector de placas fluorescentes BMG Pherastar Plus (BMG LABTECH, Ortenberg, Alemania); para la visualización geográfica de datos genotípicos se utilizó el Software KlusterCaller ${ }^{\mathrm{TM}}$ (LGC Biosearch Technologies).

\section{Mapa genético}

El ADN de los progenitores se utilizó para genotipificar toda la la población $F_{3}$ en busca de polimorfismos mediante nueve marcadores tipo SSR, cuyas temperaturas de hibridación apropiadas se obtuvieron de la base de datos GrainGenes (https://wheat.pw.usda.gov/GG3/). Los productos de las PCR se separaron en geles de acrilamida $12 \%(29: 1)$ y las bandas se detectaron usando tinción con nitrato de plata (Dreisigacker et al., 2016a).

Los resultados de los marcadores polimórficos se representaron como 0, 2, 1 y -1 para Nasma, Marroqui588, heterocigoto y dato faltante, respectivamente. Una vez sistematizados los resultados de los marcadores, se construyó un mapa genético con el software QTL IciMapping V4.1. (Li et al., 2008).

Se estableció un logaritmo mínimo de probabilidades (LOD) de 4.5 y una frecuencia máxima de recombinación de 0.30 para formar los grupos de ligamiento, mientras que la función de mapeo de Kosambi (Kosambi, 1943) se usó para convertir las frecuencias de recombinación en unidades mapa (cM):

$$
C M=1 / 4 \ln [(1+2 r) /(1-2 r)] \times 100
$$

donde r es la fracción de recombinación

\section{Análisis estadístico}

Se utilizó la prueba de ji-cuadrada $\left(\chi^{2}\right)$ para comparar la relación de segregación de las familias $F_{3}$ derivadas de plantas $F_{2}$ para dos pares de genes; cada par generó tres clases genotípicas: homocigótica dominante, segregante o heterogénea derivada de plantas $F_{2}$ heterocigóticas, y homocigótica recesiva, en una proporción esperada de $1: 2: 1$, respectivamente. Las pruebas de hipótesis fueron dos: 1) independencia entre ambos pares de genes 1:2:2:4:1:2:1:2:1 y 2) segregación 1:2:1 por separado para cada locus.

El valor de tablas y la significancia se determinó de acuerdo con la $\chi^{2}$ que se obtuvo de las proporciones de cada clase genotípica. Para el valor de tablas se usaron n-1 grados de libertad, donde $n$ es el número de clases genotípicas (Infante y Zárate, 2012); la probabilidad de la prueba de $\chi^{2}$ fue $P \leq 0.05$.

El porcentaje de recombinación se determinó con el método de Fisher y Balmukand (1928). Las familias F3 observadas se agruparon en cuatro clases: dominantedominante $\left(a_{1}\right)$, dominante-recesiva $\left(a_{2}\right)$, recesivadominante $\left(a_{3}\right)$ y doble recesiva $\left(a_{4}\right)$ (Immer y Henderson, 1943; Strickberger, 1976). Con estos datos y considerando que los genes Rht-D1 y Lr67 están en fase de repulsión debido a la mayor frecuencia de gametos parentales que recombinantes, se determinó un valor de $Z$ mediante la ecuación $\left(a_{1} \times a_{4}\right) /\left(a_{2} \times a_{3}\right)$ para posteriormente, mediante tablas, determinar el porcentaje de recombinación (Fisher y Balmukand, 1928).

\section{RESULTADOS Y DISCUSIÓN}

\section{Análisis molecular de los progenitores}

Con el marcador csLV67 se determinó la presencia del gen de resistencia a roya de la hoja $L$ r67 en el progenitor Marroqui 588 y en el control RL6077; de igual forma, se confirmó la presencia del gen de enanismo Rht-D1 en el progenitor Nasma con el marcador DF/MR2, al igual que en el control Parula, de acuerdo con los procedimientos descritos por Dreisigacker et al. (2016a) (Figura 1).

\section{Análisis de independencia}

En el Cuadro 1 se presentan las pruebas de $\chi^{2}$ para cada locus; en ambos casos, los datos genotípicos observados se ajustaron a los esperados $(P \leq 0.05)$, lo que indicó una segregación normal mendeliana. Para la segunda prueba (Cuadro 2), los resultados mostraron que ambos loci (Cuadro 2) no son independientes ( $P \leq 0.05$ ); por tanto, y combinando los resultados de ambas pruebas (Cuadros 1 
y 2), tal como lo postularon Immer y Henderson (1943) y Strickberger (1976), se concluyó que hay ligamiento entre los genes Rht-D1 y Lr67. Por los datos observados (Cuadro 2 y Figura 2), de 294 familias $F_{3}$, sólo se obtuvieron tres familias con la presencia de los genes Rht-D1 y Lr67 en el mismo genotipo y en forma homocigótica y también tres familias homocigotas con la ausencia de ambos genes; lo que indica un número relativamente bajo de estas clases recombinantes.

El valor de $Z=(156 \times 3) /(65 \times 70)$ fue de 0.1028 , mismo que en tablas de Fisher y Balmukand (1928) resulta en una frecuencia de recombinación $(r)$ de 0.22, valor que al ser introducido en la función de mapeo de Kosambi (1943) da como resultado una distancia genética de $23.6 \mathrm{cM}$.

Con $r=0.22$ y considerando la fase de repulsión entre los loci Lr67 y Rht-D1, se calcularon las proporciones esperadas de las frecuencias gaméticas cuando la frecuencia de recombinación entre dos pares de genes fue igual a 0.22 (Molina, 1992) y posteriormente se obtuvieron las frecuencias genotípicas esperadas de familias $F_{3}$ para compararse con las obtenidas (Cuadro 3). La prueba de ji-cuadrada mostró una alta concordancia $(P \leq 0.05)$ entre lo postulado con $r=0.22$ y lo obtenido con el análisis de ligamiento, lo que indicó, nuevamente, que el bajo número de familias con genotipos homocigotos recombinantes se debe a la baja frecuencia de entrecruzamiento entre los genes Lr67 y Rht-D1. Así, un posible aumento en el número de familias recombinantes homocigotas en $F_{3}$ podría lograrse aumentando el tamaño de muestra; esto es, tomando un mayor número de plantas $F_{2}$ para derivar sus correspondientes familias $F_{3}$ (Dreisigacker et al., 2016b); o bien, esperar a generaciones más avanzadas para dar oportunidad de recombinación entre los heterocigotos remanentes.

Lo anterior puede ser la razón de que en variedades modernas de trigo no se encuentren estos dos genes juntos; además, los programas de mejoramiento genético se enfocaron a incorporar el carácter de porte bajo, y como los alelos Lr67 y Rht-D1 están ligados, tal combinación difícilmente pudo presentarse en poblaciones pequeñas, por lo que ésta podría ser la razón de que haya quedado marginada del germoplasma moderno.

\section{Mapa genético de ligamiento}

De los marcadores utilizados, nueve mostraron polimorfismo en la población $F_{3}$ (Nasma/Marroqui

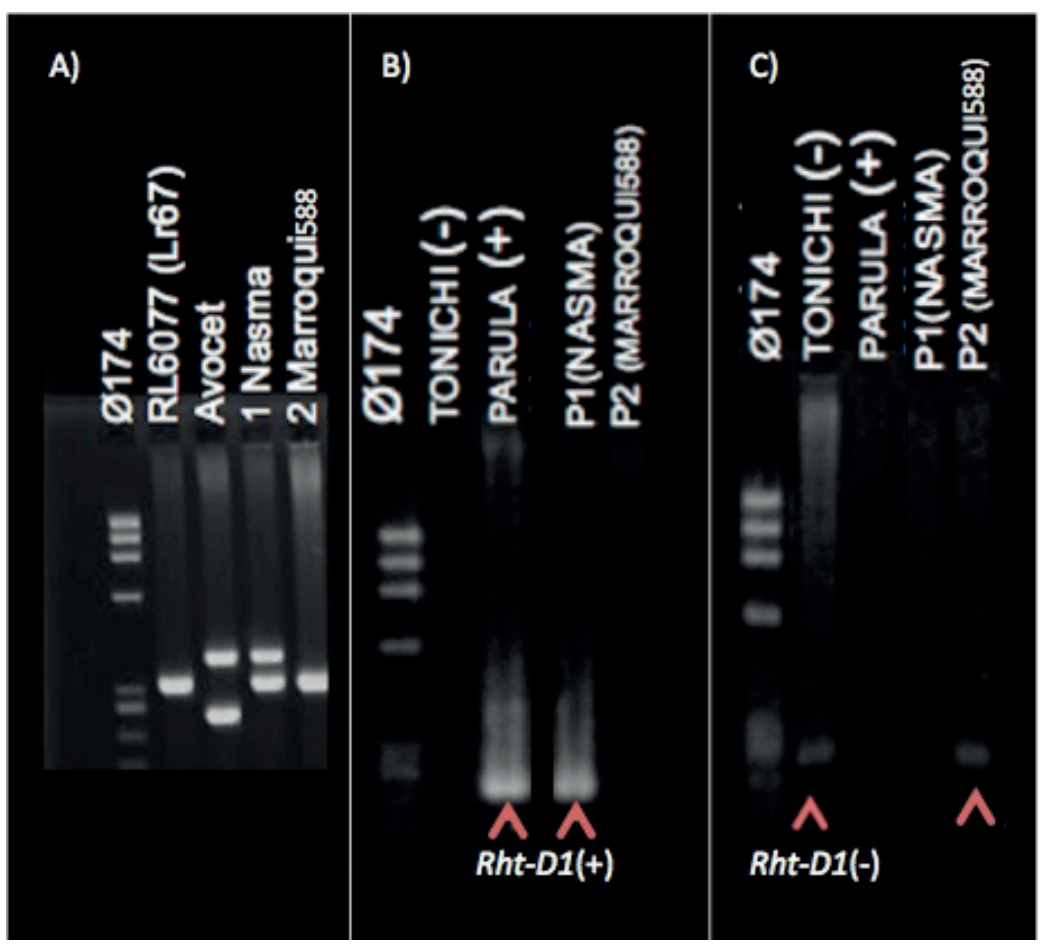

Figura 1. Amplificación de los marcadores csLV67 y DF/MR2. A) Gen Lr67, control positivo en RL6077 y en el progenitor Marroqui 588. B) Gen Rht-D1, control positivo en la variedad Parula y el progenitor Nasma. C) Complemento del marcador dominante DF/MR2 para el gen Rht-D1, control negativo en la variedad Tonichi y en el progenitor Marroqui 588. $\emptyset 174$ : marcador de peso molecular. 
Cuadro 1. Prueba de $\chi^{2}$ para los loci Rht-D1 y Lr67 por separado.

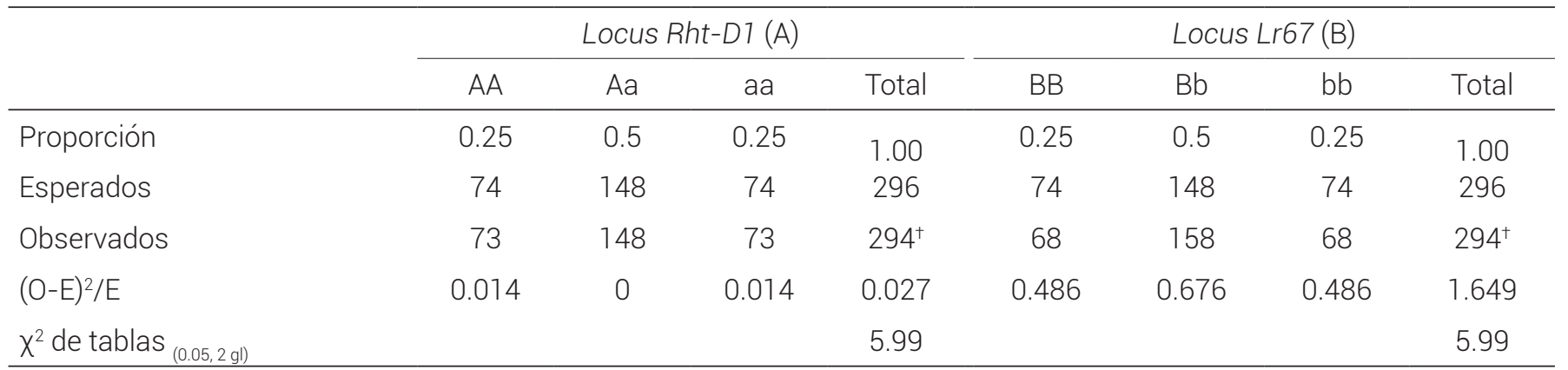

${ }^{\dagger}$ El total de los valores observados no es el mismo que el de los esperados debido a que dos muestras no amplificaron.

Cuadro 2. Prueba de $\chi 2$ para independencia entre los loci Rht-D1 (A) y Lr67 (B).

\begin{tabular}{lccccc}
\hline Genotipo & \multicolumn{2}{c}{ Proporción } & Esperados $(\mathrm{E})$ & Observados $(\mathrm{O})$ & $(\mathrm{O}-\mathrm{E})^{2} / \mathrm{E}$ \\
\hline AABB & $1 / 16$ & 0.0625 & 18.5 & 3 & 12.98 \\
$\mathrm{AABb}$ & $2 / 16$ & 0.125 & 37 & 28 & 2.18 \\
$\mathrm{AaBB}$ & $2 / 16$ & 0.125 & 37 & 17 & 10.81 \\
$\mathrm{AaBb}$ & $4 / 16$ & 0.25 & 74 & 108 & 15.62 \\
$\mathrm{AAbb}$ & $1 / 16$ & 0.0625 & 18.5 & 42 & 29.85 \\
Aabb & $2 / 16$ & 0.125 & 37 & 23 & 5.29 \\
aaBB & $1 / 16$ & 0.0625 & 18.5 & 48 & 47.04 \\
aaBb & $2 / 16$ & 0.125 & 37 & 22 & 6.08 \\
aabb & $1 / 16$ & 0.0625 & 18.5 & 3 & 12.98 \\
Total & $16 / 16$ & 1.000 & 296 & 294 & 142.86 \\
$\chi^{2}$ tablas & & & & 15.51 \\
\hline
\end{tabular}

588), cuatro SSR (wmc52, wmc720, cfd23, wmc331) y cinco SNP (BS00099053, BS00102783, BS00023258, csSNP856 y Rht-D1_SNP). De los marcadores mapeados, BS00099053 fue el que se posicionó en la parte más distal del cromosoma; a $72.17 \mathrm{cM}$ de éste se ubicó el marcador Rht-D1_SNP (ligado al gen Rht2 o Rht-D1), entre los marcadores BS00102783 y wmc331 (a 36.24 cM del primero y 20.83 cM del segundo); por su parte, el marcador csSNP856 (ligado al gen Lr67) se ubicó a 99.77 cM del marcador más distal (BS00099053), flanqueado por los marcadores wmc331 y cfd23 (a $6.77 \mathrm{cM}$ del primero y $8.16 \mathrm{cM}$ del segundo), por lo que la distancia entre los genes Rht-D1 y Lr67 fue de 27.6 cM (Figura 3).

Como mencionaron Somers et al. (2004) y Forrest et al. (2014), la baja densidad de marcadores para el cromosoma $4 \mathrm{D}$ y el bajo nivel de polimorfismo de los genotipos se ven reflejados en el desarrollo puntual de mapas de ligamiento para dicho cromosoma. En esta población de estudio se obtuvieron pocos marcadores ligados a los genes de interés, pero se logró estimar una distancia genética relativamente semejante a la reportada por Liu et al. (2014), quienes en un estudio de marcadores asociados a varios genes de interés en trigo en el cromosoma 4D reportaron una distancia aproximada de $26 \mathrm{cM}$ entre el marcador RhtD1b (SNP) y Lr67/Yr46.

\section{CONCLUSIONES}

La distancia genética entre el gen de resistencia a roya de la hoja $L$ r67 y el gen de enanismo Rht-D1, de acuerdo con el análisis de ligamiento, es de 23.6 cM y con análisis molecular, de $27.6 \mathrm{cM}$; distancia que es suficientemente grande para que exista recombinación genética entre ambos; por lo tant, no existe impedimento genético para obtener genotipos recombinantes que conjunten la resistencia a roya de la hoja ( $L$ r67) con el porte bajo de planta (Rht-D1), mismos que en los progenitores se encuentran separados y en fase de repulsión, aunque para encontrarlos es recomendable aumentar el tamaño de la población de plantas $F_{2}$ 


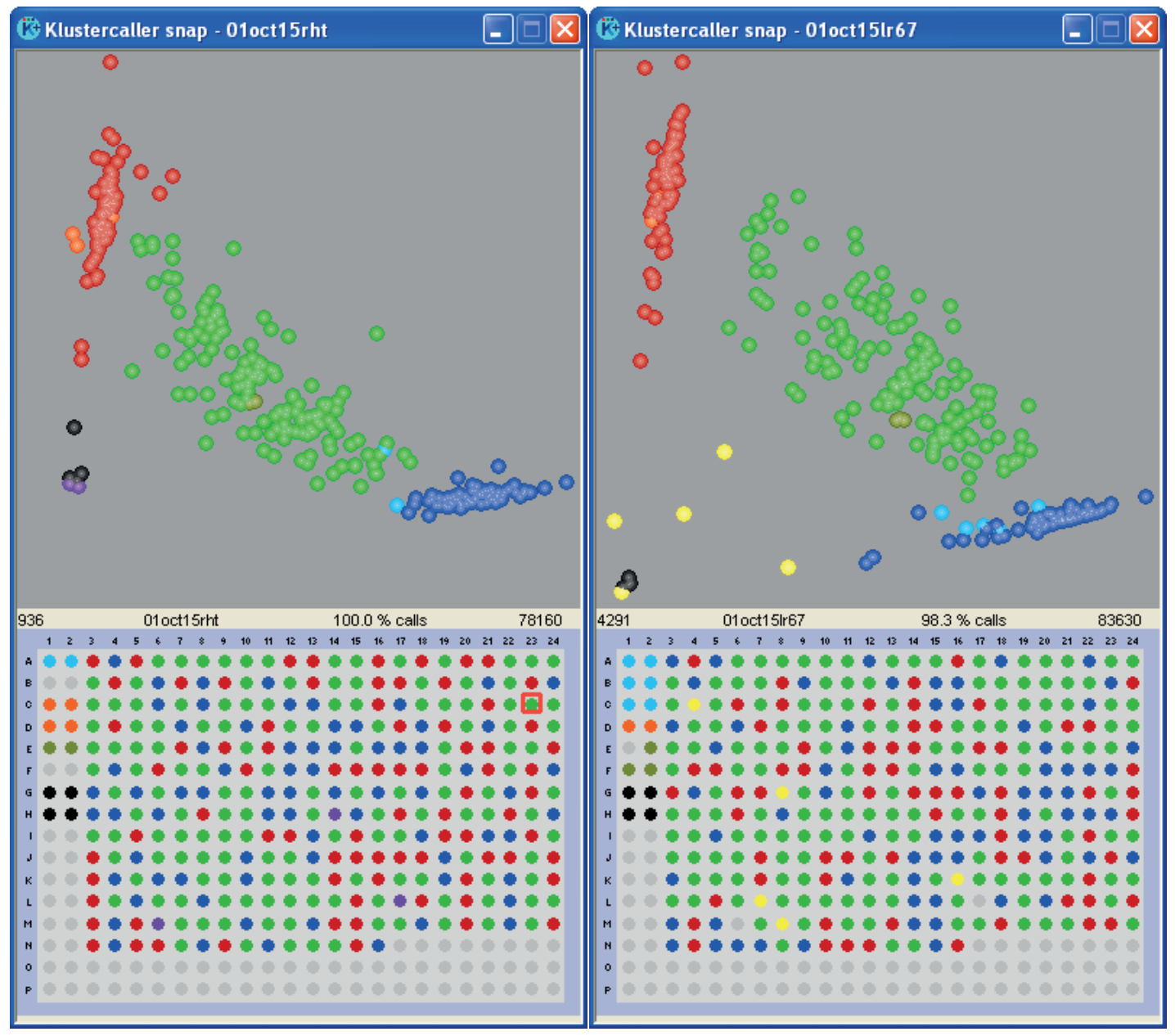

Figura 2. Visualización geográfica de datos genotípicos en una población $\mathrm{F}_{3}$ de Nasma $\times$ Marroqui 588. A) Rht-D1 (Rht-D1_ SNP); color rojo: Nasma, color azul: Marroqui 588, color verde: heterocigotos. B) Lr67 (csSNP856); color rojo: Marroqui 588, color azul: Nasma, color verde: heterocigotos.

Cuadro 3. Prueba de $\chi^{2}$ considerando una $r=0.22$ entre los loci Rht-D1 (A) y Lr67 (B).

\begin{tabular}{lcccc}
\hline Genotipos & Proporción & Esperados $(\mathrm{E})$ & Observados $(\mathrm{O})$ & $(\mathrm{O}-\mathrm{E})^{2} / \mathrm{E}$ \\
\hline $\mathrm{AABB}$ & 0.012 & 3.6 & 3 & 0.09 \\
$\mathrm{AABb}$ & 0.086 & 25.5 & 28 & 0.25 \\
$\mathrm{AaBB}$ & 0.086 & 25.5 & 17 & 2.81 \\
$\mathrm{AaBb}$ & 0.328 & 97.1 & 108 & 1.23 \\
$\mathrm{AAbb}$ & 0.152 & 45.0 & 42 & 0.20 \\
Aabb & 0.086 & 25.5 & 23 & 0.24 \\
aaBB & 0.152 & 45.0 & 48 & 0.20 \\
aaBb & 0.086 & 25.5 & 22 & 0.47 \\
aabb & 0.012 & 3.6 & 3 & 0.09 \\
Total & 1.0 & 296.0 & 294 & 5.57 \\
$\chi^{2}$ de tablas & & & 15.51 \\
\hline
\end{tabular}




\section{Cromosoma 4DL}

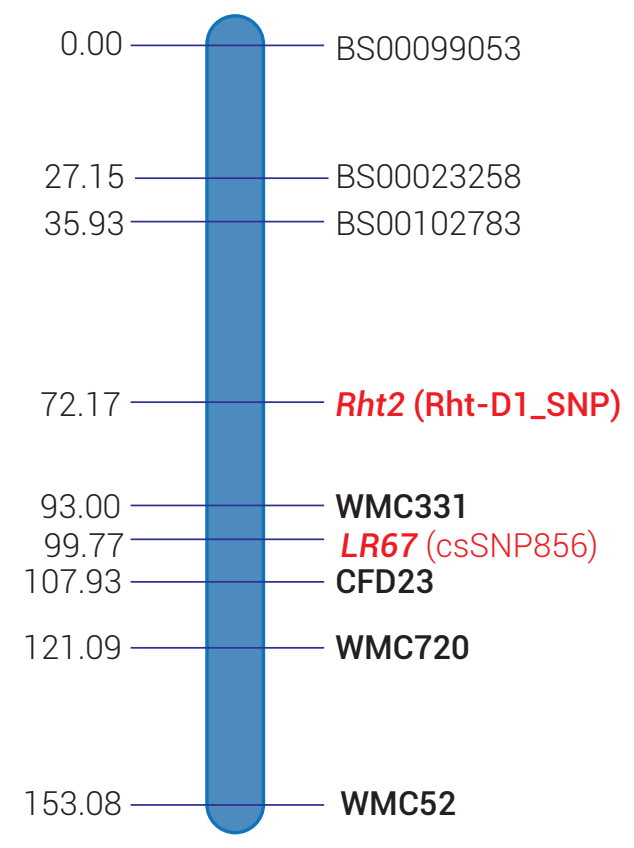

Figura 3. Mapa genético de la región del brazo largo del cromosoma 4D, usando una población $\mathrm{F}_{3}$ de Nasma/Marroqui 588, las distancias genéticas se muestran en cM. Los marcadores en color rojo indican la ubicación de los genes de interés Lr67 y Rht-D1.

\section{BIBLIOGRAFÍA}

Dreisigacker S., D. Sehgal, A. E. Reyes J., B. Luna G., S. Muñoz Z., C. Núñez R., J. Mollins and S. Mall (2016a) CIMMYT Wheat Molecular Genetics: Laboratory Protocols and Applications to Wheat Breeding. CIMMYT. Mexico, D. F. 142 p.

Dreisigacker S., S. Sukumaran, C. Guzmán, X. He, C. Lan, D. Bonnett and J. Crossa (2016b) Molecular marker-based selection tools in spring bread wheat improvement: CIMMYT experience and prospects. In: Molecular Breeding for Sustainable Crop Improvement. Sustainable Development and Biodiversity. Vol. 11. V. R. Rajpal, S. R. Rao and S. N. Raina (eds.). Springer. Cham, Switzerland. pp:421-474, https://doi.org/10.1007/978-3319-27090-6_16

Dyck P. L. and D. J. Samborski (1979) Adult-plant leaf rust resistance in PI 250413, an introduction of common wheat. Canadian Journal of Plant Science 59:329-332, https://doi.org/10.4141/cjps79053

Ellis M., W. Spielmeyer, K. Gale, G. Rebetzke and R. Richards (2002) "Perfect" markers for the Rht-B1b and Rht-D1b dwarfing genes in wheat. Theoretical and Applied Genetics 105:1038-1042, https://doi. org/10.1007/s00122-002-1048-4

Fisher R. A. and B. Balmukand (1928) The estimation of linkage from the offspring of selfed heterozygotes. Journal of Genetics 20:79-92, https://doi.org/10.1007/BF02983317

Forrest K., V. Pujol, P. Bulli, M. Pumphrey, C. Wellings, S. Herrera-Foessel, ... and W. Spielmeyer (2014) Development of a SNP marker assay for the Lr67 gene of wheat using a genotyping by sequencing approach. Molecular Breeding 34:2109-2118, https://doi. org/10.1007/s11032-014-0166-4

Herrera-Foessel S. A., E. S. Lagudah, J. Huerta-Espino, M. J. Hayden, H. S. Bariana, D. Singh and R. P. Singh (2011) New slow-rusting leaf rust and stripe rust resistance genes Lr67 and Yr46 in wheat are pleiotropic or closely linked. Theoretical and Applied Genetics 122:239-249, https://doi.org/10.1007/s00122-010-1439-x

Herrera-Foessel S. A., R. P. Singh, M. Lillemo, J. Huerta-Espino, S. Bhavani, S. Singh, C. Lan, V. Calvo-Salazar and E. S. Lagudah (2014) Lr67/Yr46 confers adult plant resistance to stem rust and powdery mildew in wheat. Theoretical and Applied Genetics 127:781-789, http:// doi.org/10.1007/s00122-013-2256-9

Hiebert C. W., J. B. Thomas, B. D. McCallum, D. G. Humphreys, R. M. DePauw, M. J. Hayden, R. Mago, W. Schnippenkoetter and W. Spielmeyer (2010) An introgression on wheat chromosome 4DL in RL6077 (Thatcher*6/PI 250413) confers adult plant resistance to stripe rust and leaf rust (Lr67). Theoretical and Applied Genetics 121:1083-1091, $\quad$ https://doi.org/10.1007/s00122-010$1373-y$

Huerta-Espino J., H. E. Villaseñor-Mir, E. Espitia-Rangel, E. Solis-Moya and M. van Ginkel (2011a) The history of wheat breeding in Mexico. In: The World Wheat Book. Vol. 2. A History of Wheat Breeding A. P. Bonjean, W. J. Angus and M. van Ginkel (eds.). Lavoisier Publishing. Paris, France. pp:275-308.

Huerta-Espino J., R. P. Singh, S. Germán, B. D. McCallum, R. F. Park, W. O Chen, S. C. Bhardwaj and H. Goyeau (2011b) Global status of wheat leaf rust caused by Puccinia triticina. Euphytica 179:143-160, https://doi.org/10.1007/s10681-011-0361-x

Immer F. R. and M. T. Henderson (1943) Linkage studies in barley. Genetics 28:419-440.

Infante G. S. y G. P. Zárate L. (2012) Métodos Estadísticos. Un Enfoque Interdisciplinario. Tercera edición. Mundi-Prensa. México, D. F. $624 \mathrm{p}$.

Jlibene M. and N. Nsarellah (2011) Wheat breeding in Morocco, a historical perspective. In: The World Wheat Book. Vol. 2. A History of Wheat Breeding. A. P. Bonjean, W. J. Angus and M. van Ginkel (eds.). Lavoisier Publishing. Paris, France. pp:425-442.

Kosambi D. D. (1943) The estimation of map distances from recombination values. Annals of Eugenics 12:172-175, https:// doi.org/10.1111/j.1469-1809.1943.tb02321.x

Li H., J. M. Ribaut, Z. Li and J. Wang (2008) Inclusive composite interval mapping (ICIM) for digenic epistasis of quantitative traits in biparental populations. Theoretical and Applied Genetics 116:243-260, https://doi.org/10.1007/s00122-007-0663-5

Li H., S. Singh, S. Bhavani, R. P. Singh, D. Sehgal, B. R. Basnet, P. Vikram, J. Burgueno-Ferreira and J. Huerta-Espino (2016) Identification of genomic associations for adult plant resistance in the 
background of popular south Asian wheat cultivar, PBW343. Frontiers in Plant Science 7:1674, https://doi.org/10.3389/ fpls.2016.01674

Liu S., J. C. Rudd, G. Bai, S. D. Haley, A. M. H. Ibrahim, Q. Xue, ... and P. S. Amand (2014) Molecular markers linked to important genes in hard winter wheat. Crop Science 54:1304-1321, https://doi. org/10.2135/cropsci2013.08.0564

Molina G. J. D. (1992) Introducción a la Genética de Poblaciones y Cuantitativa (Algunas Implicaciones en Genotecnia). AGT Editor. México, D. F. 349 p.

Moore J. W., S. Herrera-Foessel, C. Lan, W. Schnippenkoetter, M. Ayliffe, J. Huerta-Espino, ... and E. Lagudah (2015) A recently evolved hexose transporter variant confers resistance to multiple pathogens in wheat. Nature Genetics 47:1494-1498, https://doi.org/10.1038/ ng.3439

Niederhauser J. S., J. Cervantes and L. Servin (1954) Late blight in Mexico and its implications. Phytopathology 44:406-408.

Rodríguez-García M. F., R. I. Rojas-Martínez, J. Huerta-Espino, H. E. VillaseñorMir, E. Zavaleta-Mejía, J. S. Sandoval-Islas y J. F. Crossa-Hiriart (2019) Genética de la resistencia a roya amarilla causada por Puccinia striiformis f. sp. tritici W. en tres genotipos de trigo (Triticum aestivum L.). Revista Fitotecnia Mexicana 42:31-38, https://doi. org/10.35196/rfm.2019.1.31-38

Sandoval-Sánchez M., J. Huerta-Espino, R. P. Singh, M. Singh-Randhawa,
R. I. Rojas-Martínez, I. Benitez-Riquelme y C. Nava-Díaz (2019) Resistencia parcial y específica a roya del tallo en la línea avanzada de trigo harinero 'Kijil'. Revista Fitotecnia Mexicana 42:411-418, https://doi.org/10.35196/rfm.2019.4.411-418

Sayre K. D., S. Rajaram and R. A. Fischer (1997) Yield potential progress in short bread wheats in Northwest Mexico. Crop Science 37:3642, https://doi.org/10.2135/cropsci1997.0011183X003700010 $006 x$

Singh R. P., J. Huerta-Espino and S. Rajaram (2000) Achieving nearimmunity to leaf and stripe rusts in wheat by combining slow rusting resistance genes. Acta Phytopathologica et Entomologica Hungarica 35:133-139.

Singh R. P., P. K. Singh, J. Rutkoski, D. P. Hodson, X. He, L. N. Jørgensen, M. S Hovmøller and J. Huerta-Espino (2016) Disease impact on wheat yield potential and prospects of genetic control. Annual Review of Phytopathology 54:303-322, https://doi.org/10.1146/ annurev-phyto-080615-095835

Somers D. J., P. Isaac and K. Edwards (2004) A high-density microsatellite consensus map for bread wheat (Triticum aestivum L.) Theoretical and Applied Genetics 109:1105-1114, https://doi. org/10.1007/s00122-004-1740-7

Strickberger M. W. (1976) Genetics. Macmillan Publishers. London, UK. $847 \mathrm{p}$. 